SUPPORTING INFORMATION

\title{
Ultrasensitive Electrochemistry by Radical \\ Annihilation Amplification in a Solid-Liquid Microgap
}

Rezvan Kazemi ${ }^{\mathrm{a}}$, Nicole E. Tarolla ${ }^{\mathrm{a}}$, Jeffrey E. Dick ${ }^{\mathrm{a}, \mathrm{b} *}$

aDepartment of Chemistry, The University of North Carolina at Chapel Hill, Chapel Hill, NC 27599, USA

bLineberger Comprehensive Cancer Center, School of Medicine, The University of North Carolina at Chapel Hill, Chapel Hill, NC 27599, USA

To whom correspondence should be addressed: jedick@email.unc.edu 


\section{Table of contents}

Section

Contents

Page

1 Voltammetric Characterizations of DmFc and Oxalate on Pt UME and CF UMEs (Figure S1)

2 Probing the Solubility of $\mathrm{DmFC}^{+}$in Toluene Phase

3 DLS Results for Toluene|Water Emulsion (100 $\mu \mathrm{L}: 5 \mathrm{~mL}, \mathrm{v} / \mathrm{v})$ (Figure S2)

4 DLS Results for Toluene|Water Emulsion (1 mL:5 mL, v/v) (Figure S3)

5 Finite-Element Simulation of RAA Mechanism in a Microdroplet Emulsion

S7-9 System

6 References

S-10

7 COMSOL Report

S11-21 


\section{Voltammetric Characterizations of DmFc and Oxalate on a Pt UME}
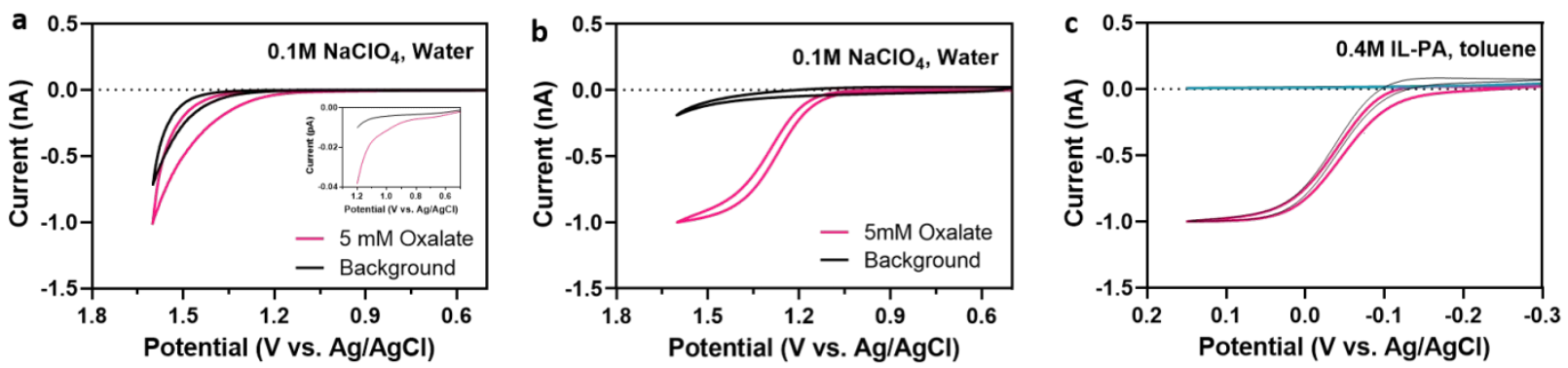

Figure S1. Cyclic voltammograms showing the oxidation of $5 \mathrm{mM}$ sodium oxalate on a) platinum ultramicroelectrode b) carbon fiber ultramicroelectrode c) cyclic voltammograms of $1 \mathrm{mM} \mathrm{DmFc}$ on a platinum (pink line) and carbon fiber ultramicroelectrodes (black line) in the indicated solvents at a scan rate of $0.1 \mathrm{~V} / \mathrm{s}$ (the teal line displays the cyclic voltammogram of background). Based on these voltammograms, an applied potential of $+0.8 \mathrm{~V}$ and $+1.15 \mathrm{~V}$ vs Ag/AgCl were chosen for the $i$-t experiments on a platinum and carbon fiber ultramicroelectrodes, respectively. The applied potential of $+0.8 \mathrm{~V}$ is sufficient for mass transfer limited oxidation of DmFc in the toluene phase and kinetic limited oxidation of oxalate in the continuous phase without interference of water or electrode oxidation in the RAA mechanism. 


\section{Probing the Solubility of $\mathrm{DmFc}^{+}$in Toluene Phase}
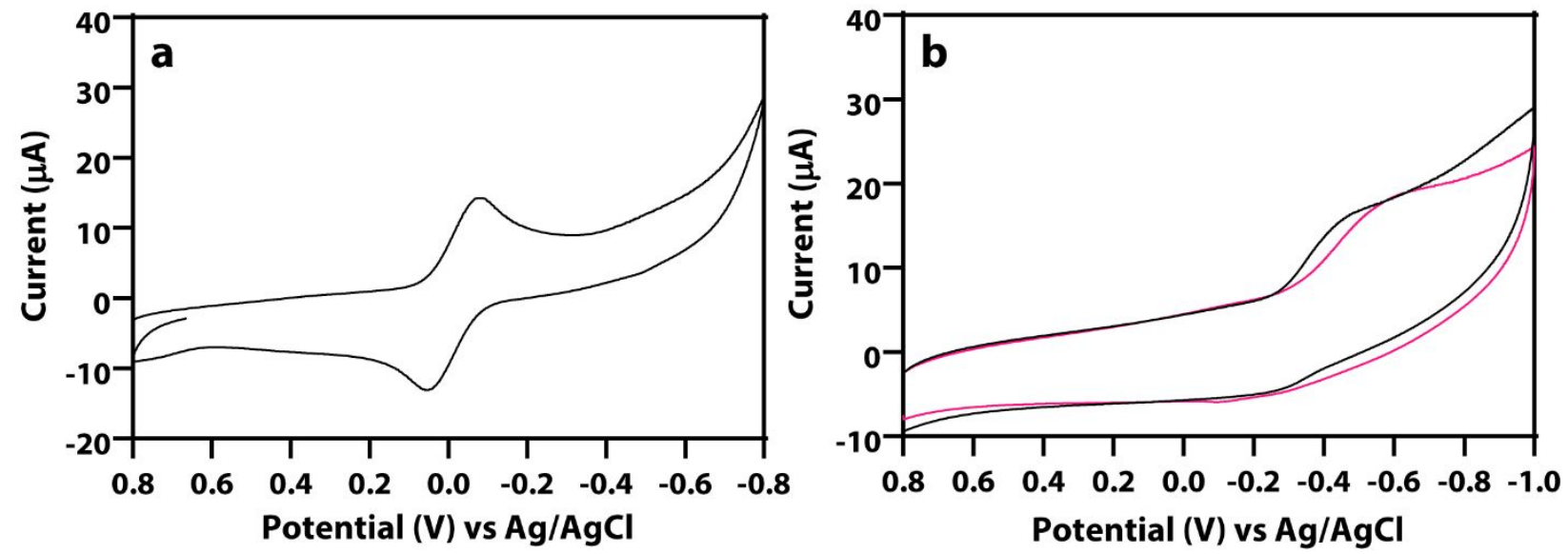

Figure S2. a) Cyclic voltammogram of $1 \mathrm{mM} \mathrm{DmFc}^{+}$after being generated by bulk electrolysis in toluene b) cyclic voltammograms of $0.1 \mathrm{M} \mathrm{KCl}$ on a glassy carbon macroelectrode before (black line) and after (pink line) mixing with toluene solution containing $\mathrm{DmFc}^{+}$(black line) at a scan rate of $0.1 \mathrm{~V} / \mathrm{s}$. Figure $\mathrm{b}$ indicates that $\mathrm{DmFc}^{+}$species is not soluble in the aqueous phase. 


\section{DLS results for the Toluene|Water Emulsion ( $100 \mu \mathrm{L}: 5 \mathrm{~mL}, \mathrm{v} / \mathrm{v})$}

Because the electrochemical current spikes were collected over 1000 second $i$-t measurements, the DLS size distribution of the emulsions in the absence and presence of oxalate were monitored over the same period at three consecutive time intervals: 0-330 sec, 330-660 sec, and 660-1000 sec.

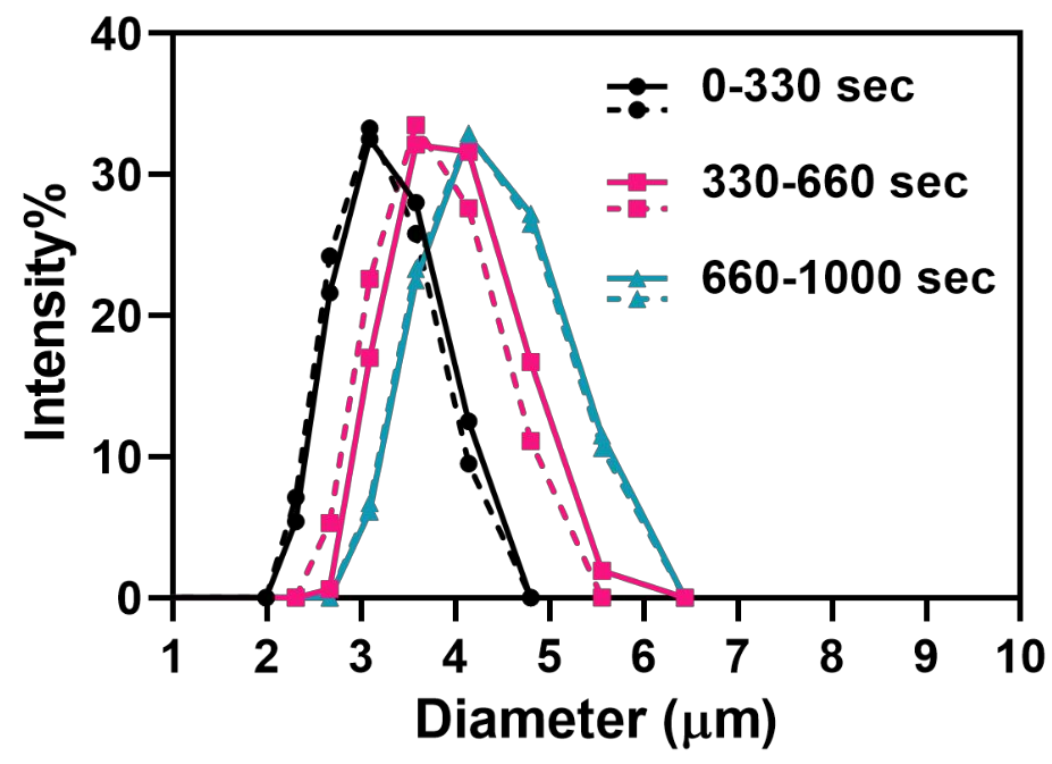

Figure S3. DLS size distributions for the toluene/water emulsion. $100 \mu \mathrm{L}$ of the toluene phase was added in $5 \mathrm{~mL}$ of the aqueous phase containing $10 \mathrm{mM} \mathrm{NaClO}_{4}$. The dashed curves are in the absence of oxalate and the solid curves are in the presence $10 \mu \mathrm{M}$ oxalate. 
4. DLS results for the Toluene|Water Emulsion ( $1 \mathrm{~mL}: 5 \mathrm{~mL}, \mathrm{v} / \mathrm{v})$

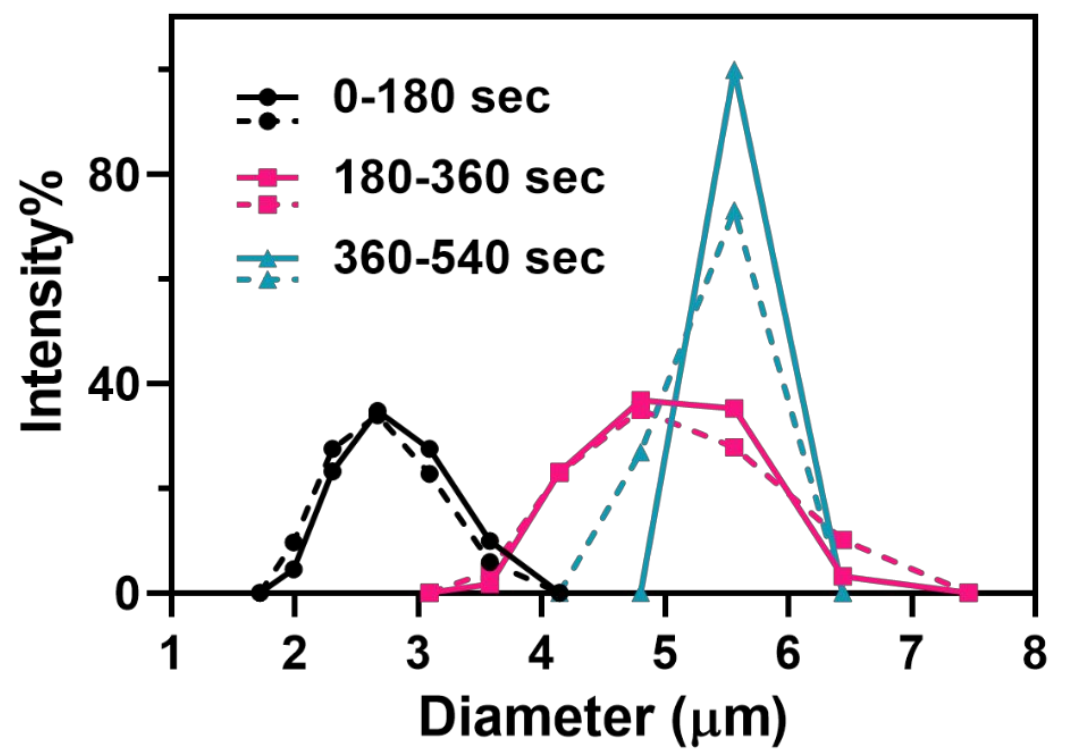

Figure S4. DLS size distributions for the toluene|water emulsion. $1 \mathrm{~mL}$ of the toluene phase was added in $5 \mathrm{~mL}$ of the aqueous phase containing $10 \mathrm{mM} \mathrm{NaClO}_{4}$. The dashed curves are in the absence of sodium oxalate and the solid curves are in the presence $10 \mu \mathrm{M}$ oxalate. 


\section{Section 5. Finite-Element Simulation of the RAA Mechanism in a Microdroplet Emulsion System:}

The $i$-t response of a single droplet collided on an electrode was simulated using finite-element modeling. The temporal and spatial concentrations of $\mathrm{DmFc}$ and $\mathrm{DmFc}^{+}$were modeled within the droplet with the diffusional transport governed by Fick's law.

The oxidation of the $\mathrm{DmFc} / \mathrm{DmFc}^{+}$couple inside the toluene droplet can be described by Equation 1 with the electron transfer reaction at the electrode/toluene droplet interface governed by Butler-Volmer kinetics.

$$
D m F c-e^{-} \rightleftarrows D m F c^{+} \#(1)
$$

The concurrent oxidation of oxalate in the aqueous phase is a multistep reaction ${ }^{1}$ that was simplified as Equation 2 with the electron transfer reaction at the electrode/aqueous solution interface governed by Butler-Volmer kinetics.

$$
\mathrm{C}_{2} \mathrm{O}_{4}^{2-}-e^{-} \rightleftarrows \mathrm{CO}_{2}^{-} \#(2)
$$

Additionally, at the interface of the toluene droplet and the bulk aqueous phase, the partitioning of the $\mathrm{CO}_{2}{ }^{-}$ species into the toluene phase can be expressed as a phase transfer reaction with the rate constant of $k_{p t}$ as shown below:

$$
\mathrm{CO}_{2}^{-}{ }_{\mathrm{Aq}} \rightleftarrows \mathrm{CO}_{2}^{-}{ }_{\text {org }} \mid k_{p t}=0.1 \mathrm{~ms}^{-1}, \mathrm{P}_{\mathrm{CO}_{2}^{-}}=1 \#(3)
$$

For simplicity, we considered a non-preferential partitioning coefficient $(P)$ of 1 for $\mathrm{CO}_{2}^{-}$, indicating that as much as the equilibrium favors radical transfer into the aqueous phase it equally favors transferring the radical into the organic phase. The transferred $\mathrm{CO}_{2}{ }^{-}$radicals inside the droplet regenerate $\mathrm{DmFc}$ molecules by reacting with $\mathrm{DmFc}^{+}$species via a radical annihilation reaction. This reaction was described with the reaction rate, $k_{\text {anni } r x n},{ }^{1}$ as:

$$
\mathrm{DmFC}^{+}+\mathrm{CO}_{2}^{-} \rightarrow \mathrm{DmFC} \mid k_{\text {anni } r x n}=6 \times 10^{9} \mathrm{M}^{-1} \mathrm{~S}^{-1} \#(4)
$$

To test the effect of the wetting properties of droplets on the electrode surface, the adsorbed droplet was modeled with two different geometries: sphere and ellipsoid with the same volume being preserved for the two geometries. The spherical droplet was modeled as a segment of a sphere intersecting with the electrode surface with a contact radius $1 / 5^{\text {th }}$ of the sphere radius. The ellipsoidal toluene droplet was modeled as a half of an ellipsoid intersecting with the electrode surface where the contact radius equals the ellipsoid radius. The bulk aqueous phase extended to 50 times the radius of the droplet. Figure S5a and S5b show a representation of the 2D axisymmetric COMSOL model used to calculate the $i$-t response of a droplet collision along with the mesh used in the discretization of the model for spherical and ellipsoidal geometries, respectively. 


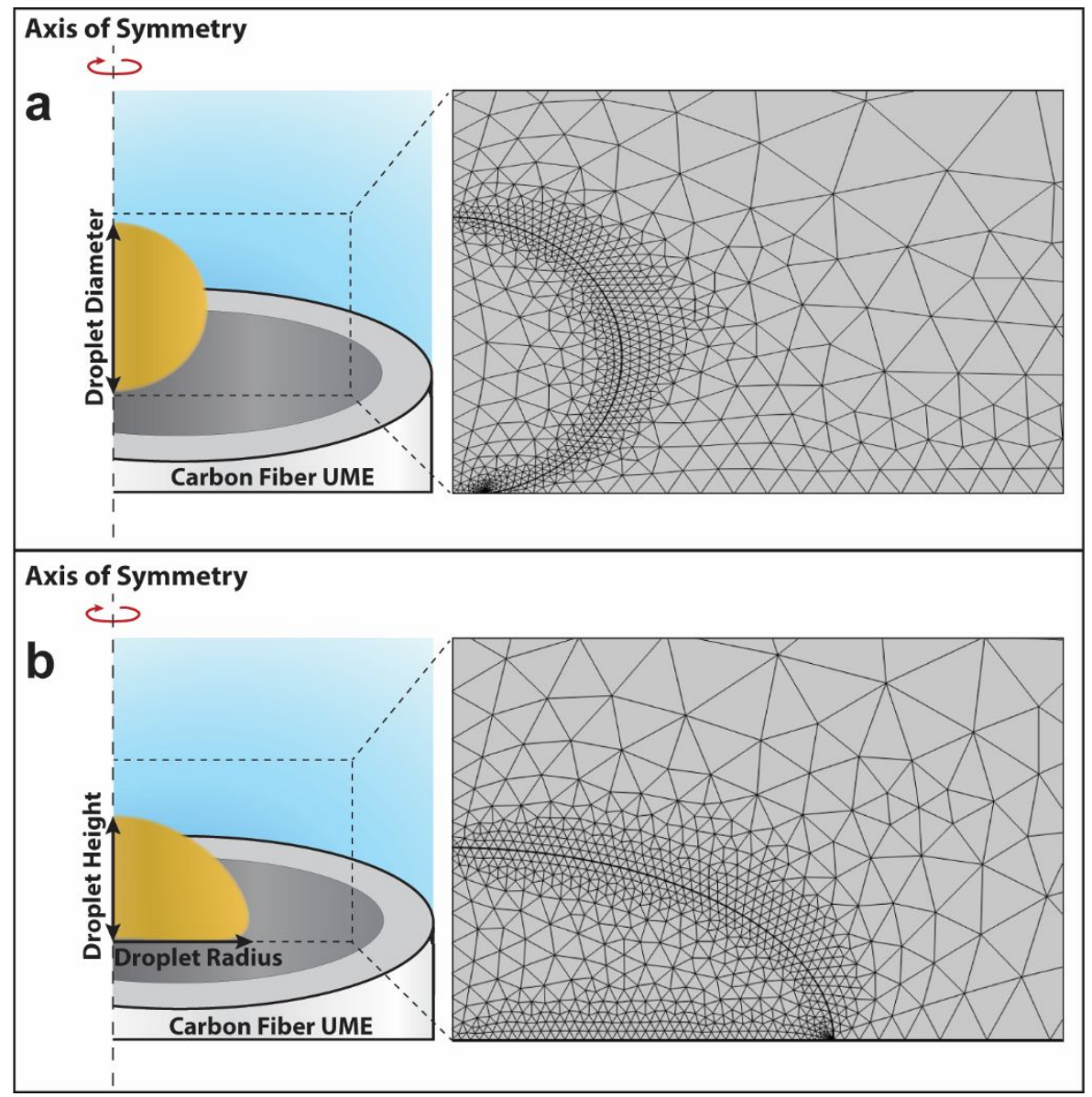

Figure S5. Schematic demonstrating the 2D axisymmetric COMSOL model used for the simulation of the i-t response of a single droplet. The vertical dashed line represents the axis of symmetry with the right panels showing a magnified view of the FiniteElement mesh applied in the model for the droplet geometry of a) sphere b) ellipsoid. 
All simulations were conducted using the Transport of Dilute Species Module within COMSOL 5.5. Computation was completed on a supercomputing cluster on a single node using 4 physical cores on 2.50 $\mathrm{GHz}$ Intel processors. Additional simulation details are available upon request.

Table S1. Relevant COMSOL Parameters.

\begin{tabular}{|c|c|c|c|}
\hline Parameter & Value & Units & Description \\
\hline $\mathrm{cDmFc}$ & $5 \times 10^{-5}$ & $\mathrm{~mol} / \mathrm{m}^{3}$ & Initial concentration of $\mathrm{DmFc}$ in organic phase \\
\hline cDmFcP & 0 & $\mathrm{~mol} / \mathrm{m}^{3}$ & Initial concentration of $\mathrm{DmFc}^{+}$in organic phase \\
\hline $\mathrm{cOx}$ & 0.01 & $\mathrm{~mol} / \mathrm{m}^{3}$ & Initial concentration of oxalate in aqueous phase \\
\hline cRad_Aq & 0 & $\mathrm{~mol} / \mathrm{m}^{3}$ & Concentration of $\mathrm{CO}_{2}{ }^{-}$radical in aqueous phase \\
\hline cRad_Org & 0 & $\mathrm{~mol} / \mathrm{m}^{3}$ & Concentration of $\mathrm{CO}_{2}{ }^{-}$radical in organic phase \\
\hline $\mathrm{dDmFc}$ & $2.43 \times 10^{-9}$ & $\mathrm{~m}^{2} / \mathrm{s}$ & Diffusion coefficient of DmFc in organic phase \\
\hline $\mathrm{dDmFcP}$ & $2.43 \times 10^{-9}$ & $\mathrm{~m}^{2} / \mathrm{s}$ & Diffusion coefficient of $\mathrm{DmFc}^{+}$in organic phase \\
\hline $\mathrm{dOx}$ & $1 \times 10^{-9}$ & $\mathrm{~m}^{2} / \mathrm{s}$ & Diffusion coefficient of oxalate in in aqueous phase \\
\hline dRad_Org & $1 \times 10^{-9}$ & $\mathrm{~m}^{2} / \mathrm{s}$ & Diffusion coefficient of $\mathrm{CO}_{2}{ }^{-}$radical in in organic phase \\
\hline $\mathrm{dRad} / \mathrm{Aq}$ & $1 \times 10^{-9}$ & $\mathrm{~m}^{2} / \mathrm{s}$ & Diffusion coefficient of $\mathrm{CO}_{2}{ }^{-}$radical in in aqueous phase \\
\hline EFml_Ox & 0.85 & $0.85 \mathrm{~V}$ & Formal potential of $\mathrm{C}_{2} \mathrm{O}_{4}{ }^{2-} / \mathrm{C}_{2} \mathrm{O}_{4}-$ couple in aqueous phase \\
\hline EFml_DmFc & -0.043 & $-0.043 \mathrm{~V}$ & Formal potential for $\mathrm{DmFc} / \mathrm{DmFc}^{+}$couple in toluene phase \\
\hline EAppl & 1.15 & $1.15 \mathrm{~V}$ & Applied potential \\
\hline k0_Ox & 0.01 & $\mathrm{~m} / \mathrm{s}$ & Heter. rate constant for oxalate oxidation \\
\hline $\mathrm{k} 0 \_\mathrm{DmFc}$ & 0.01 & $\mathrm{~m} / \mathrm{s}$ & Heter. rate constant for DmFc oxidation \\
\hline $\mathrm{k} \mathrm{pt}$ & 0.1 & $\mathrm{~m} / \mathrm{s}$ & Phase transfer rate constant \\
\hline $\mathrm{k}$ anni $\mathrm{rxn}$ & $6 \times 10^{9}$ & $\mathrm{~m}^{3} /(\mathrm{s} \cdot \mathrm{mol})$ & Annihilation reaction rate constant \\
\hline P_Rad & 1 & & $\mathrm{CO}_{2}{ }^{-{ }_{\mathrm{Aq}}} / \mathrm{CO}_{2}{ }^{-}{ }_{\mathrm{Org}}$ Partition Coefficient \\
\hline $\mathrm{F}$ & 96485 & $\mathrm{C} / \mathrm{mol}$ & Faraday constant \\
\hline alpha & 0.5 & & Transfer Coefficient \\
\hline
\end{tabular}




\section{References}

1. Kai, T.; Zhou, M.; Johnson, S.; Ahn, H. S.; Bard, A. J., Direct Observation of C2O4-- and CO2by Oxidation of Oxalate within Nanogap of Scanning Electrochemical Microscope. J. Am. Chem. Soc. 2018, 140 (47), 16178-16183. 
Section 7. COMSOL Report

MULTIPHYSSICS D

\section{Ellipsoidal geometry for $50 \mathrm{nM}$ droplet experiment}

\section{Report date}

Nov 3, 2020 10:00:14 AM 


\section{Global Definitions}

Date Sep 10, 2020 9:53:46 AM

\section{GLOBAL SETTINGS}

USED PRODUCTS

COMSOL Multiphysics

Chemical Reaction Engineering Module

\subsection{PARAMETERS}

\section{PARAMETERS 1}

\begin{tabular}{|c|c|c|c|}
\hline Name & Expression & Value & Description \\
\hline $\mathrm{T}$ & $293[K]$ & $293 \mathrm{~K}$ & Temperature \\
\hline rElect & $5 e-06[\mathrm{~m}]$ & $5 \mathrm{E}-6 \mathrm{~m}$ & Electrode radius \\
\hline rDrop & $2 e-06[\mathrm{~m}]$ & $2 \mathrm{E}-6 \mathrm{~m}$ & Droplet radius \\
\hline R2 & $8.314[\mathrm{~J} / \mathrm{K} / \mathrm{mol}]$ & $8.314 \mathrm{~J} /(\mathrm{mol} \cdot \mathrm{K})$ & Gas constant \\
\hline PC_Rad & 1 & 1 & $\begin{array}{l}\text { partition coefficient for } \\
\text { Radical(cAq/cOrg) }\end{array}$ \\
\hline $\mathrm{nOx}$ & 1 & 1 & \# of e for oxalate oxidation \\
\hline $\mathrm{nCp}$ & 1 & 1 & \# of e for CP* oxidation \\
\hline nAnni & 1 & 1 & \# of e for annihilation \\
\hline kO_Ox & $1[\mathrm{~cm} / \mathrm{s}]$ & $0.01 \mathrm{~m} / \mathrm{s}$ & $\begin{array}{l}\text { Heter. rate constant or oxalate } \\
\text { oxidation }\end{array}$ \\
\hline kO_Cp & $1[\mathrm{~cm} / \mathrm{s}]$ & $0.01 \mathrm{~m} / \mathrm{s}$ & Heter. rate constant or $\mathrm{CP}$ oxidation \\
\hline k_pt & $10[\mathrm{~cm} / \mathrm{s}]$ & $0.1 \mathrm{~m} / \mathrm{s}$ & $\begin{array}{l}\text { phase transfer rate of Rad from Aq to } \\
\text { Org }\end{array}$ \\
\hline k_anni & $6 e 09[1 / \mathrm{M} / \mathrm{s}]$ & $6 \mathrm{E} 6 \mathrm{~m}^{3} /(\mathrm{s} \cdot \mathrm{mol})$ & Annihilation rate constant \\
\hline hDrop & $0.5 *$ rDrop & $1 \mathrm{E}-6 \mathrm{~m}$ & \\
\hline $\mathrm{F}$ & $96485[\mathrm{C} / \mathrm{mol}]$ & $96485 \mathrm{C} / \mathrm{mol}$ & Faraday constant \\
\hline $\mathrm{f}$ & $\mathrm{F} /(\mathrm{R} 2 * \mathrm{~T})$ & $39.6081 / \mathrm{V}$ & reduced Faraday's \\
\hline EFml_Ox & $0.85[\mathrm{~V}]$ & $0.85 \mathrm{~V}$ & Formal potential of C2O4/C2O4.- \\
\hline EFml_Cp & $-0.043[\mathrm{~V}]$ & $-0.043 \mathrm{~V}$ & Formal potential of $\mathrm{CP} / \mathrm{CP}+$ \\
\hline EAppl & $1.15[\mathrm{~V}]$ & $1.15 \mathrm{~V}$ & Applied potential \\
\hline dRad_Org & $1 \mathrm{e}-05\left[\mathrm{~cm}^{\wedge} 2 / \mathrm{s}\right]$ & $1 \mathrm{E}-9 \mathrm{~m}^{2} / \mathrm{s}$ & diffusion coefficient of radical in Org \\
\hline dRad_Aq & $1 \mathrm{e}-05\left[\mathrm{~cm}^{\wedge} 2 / \mathrm{s}\right]$ & $1 \mathrm{E}-9 \mathrm{~m}^{2} / \mathrm{s}$ & diffusion coefficient of radical in $\mathrm{Aq}$ \\
\hline$d O x$ & $1 \mathrm{e}-05\left[\mathrm{~cm}^{\wedge} 2 / \mathrm{s}\right]$ & $1 \mathrm{E}-9 \mathrm{~m}^{2} / \mathrm{s}$ & diffusion coefficient of oxalate in $\mathrm{Aq}$ \\
\hline dCp_red & $2.43 e-05\left[\mathrm{~cm}^{\wedge} 2 / \mathrm{s}\right]$ & $2.43 \mathrm{E}-9 \mathrm{~m}^{2} / \mathrm{s}$ & diffusion coefficient of DmFc+ \\
\hline$d C p$ & $2.43 e-05\left[\mathrm{~cm}^{\wedge} 2 / \mathrm{s}\right]$ & $2.43 \mathrm{E}-9 \mathrm{~m}^{2} / \mathrm{s}$ & diffusion coefficient of $\mathrm{DmFc}$ \\
\hline cRad_Org & $0 \mathrm{e}-06[\mathrm{~mol} / \mathrm{L}]$ & $0 \mathrm{~mol} / \mathrm{m}^{3}$ & Concentration of radical in Org \\
\hline cRad_Aq & $0 \mathrm{e}-06[\mathrm{~mol} / \mathrm{L}]$ & $0 \mathrm{~mol} / \mathrm{m}^{3}$ & Concentration of radical in $\mathrm{Aq}$ \\
\hline
\end{tabular}




\begin{tabular}{|l|l|l|l|}
\hline Name & Expression & Value & Description \\
\hline cOx & $10 \mathrm{e}-06[\mathrm{~mol} / \mathrm{L}]$ & $0.01 \mathrm{~mol} / \mathrm{m}^{3}$ & concentration of oxalate \\
\hline cCp_red & $0 e-06[\mathrm{~mol} / \mathrm{L}]$ & $0 \mathrm{~mol} / \mathrm{m}^{3}$ & Concentration of DmFc+ \\
\hline cCp & $50 \mathrm{e}-09[\mathrm{~mol} / \mathrm{L}]$ & $5 \mathrm{E}-5 \mathrm{~mol} / \mathrm{m}^{3}$ & Concentration of $\mathrm{DmFc}$ \\
\hline alpha & 0.5 & 0.5 & Transfer Coefficient \\
\hline
\end{tabular}




\section{Component 1}

\subsection{DEFINITIONS}

\subsubsection{Coordinate Systems}

Boundary System 1

\begin{tabular}{|l|l|}
\hline Coordinate system type & Boundary system \\
\hline Tag & sys1 \\
\hline
\end{tabular}

COORDINATE NAMES

First Second Third

$\mathrm{t} 1$ to $\mathrm{n}$

\subsection{GEOMETRY 1}

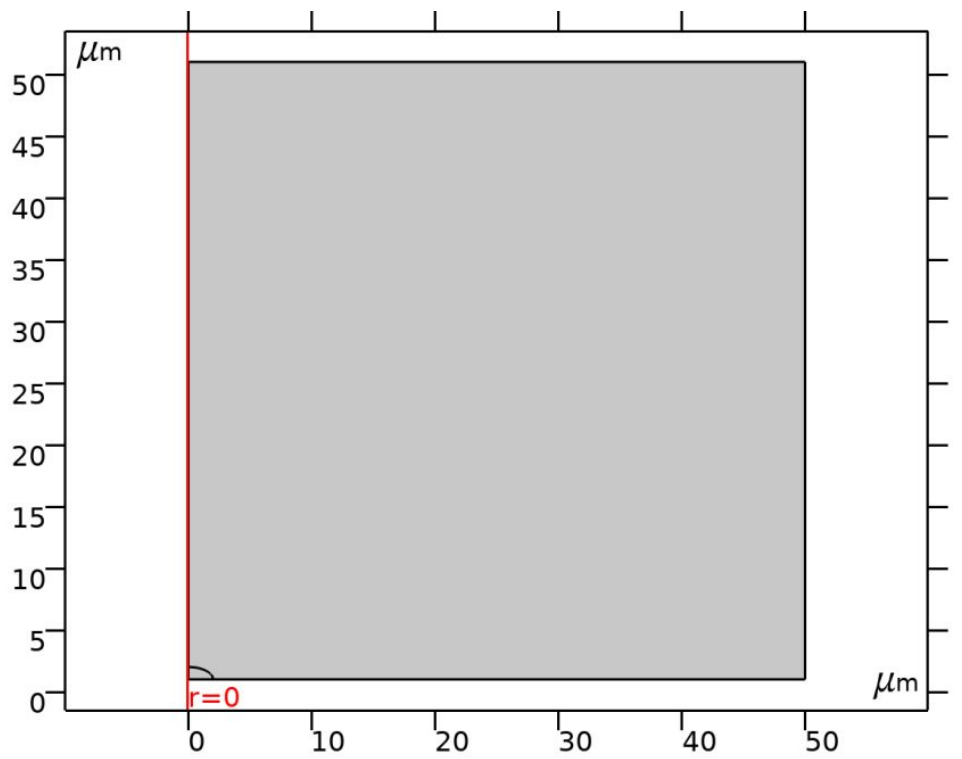

Geometry 1

UNITS

Length unit $\mu \mathrm{m}$

Angular unit deg 


\subsection{TRANSPORT OF DILUTED SPECIES AQUEOUS}

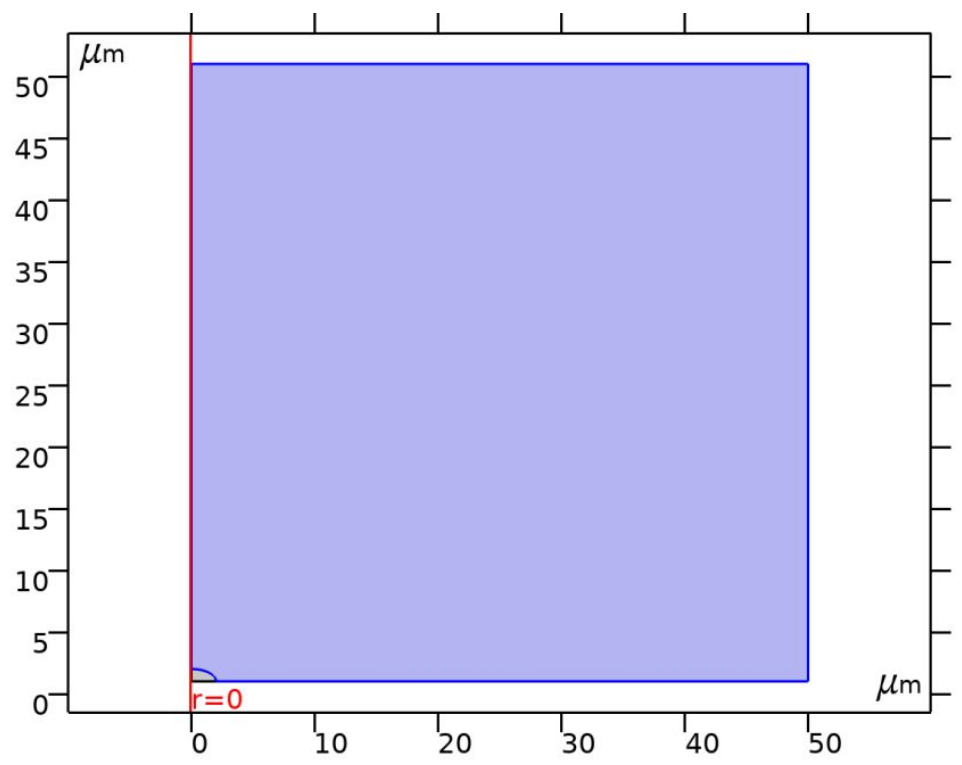

Transport of Diluted Species Aqueous

EQUATIONS

$$
\begin{aligned}
& \frac{\partial c_{i}}{\partial t}+\nabla \cdot \mathbf{J}_{i}=R_{i} \\
& \mathbf{J}_{i}=-D_{i} \nabla c_{i}
\end{aligned}
$$

\section{FEATURES}

Transport Properties Aq

Axial Symmetry 1

No Flux 1

Initial Values 1

bulk Concentration

Aq Electrode flux

Flux_phase transfer

\subsubsection{Transport Properties Aq}

EQUATIONS

$$
\begin{aligned}
& \frac{\partial c_{i}}{\partial t}+\nabla \cdot \mathbf{J}_{i}=R_{i} \\
& \underset{\mathbf{j}_{i}=-D_{i} \nabla c_{i}}{ }
\end{aligned}
$$

\subsubsection{No Flux 1}

EQUATIONS

$$
-\mathbf{n} \cdot \mathbf{J}_{i}=0
$$




\subsection{3 bulk Concentration}

EQUATIONS

$c_{i}=c_{0, j}$

\subsubsection{Aq Electrode flux}

EQUATIONS

$\mathbf{- n} \cdot \mathbf{J}_{i}=\jmath_{0, j}$

\subsubsection{Flux_phase transfer}

EQUATIONS

$$
-\mathbf{n} \cdot J_{i}=J_{0, j}
$$

\subsection{TRANSPORT OF DILUTED SPECIES DROPLET}

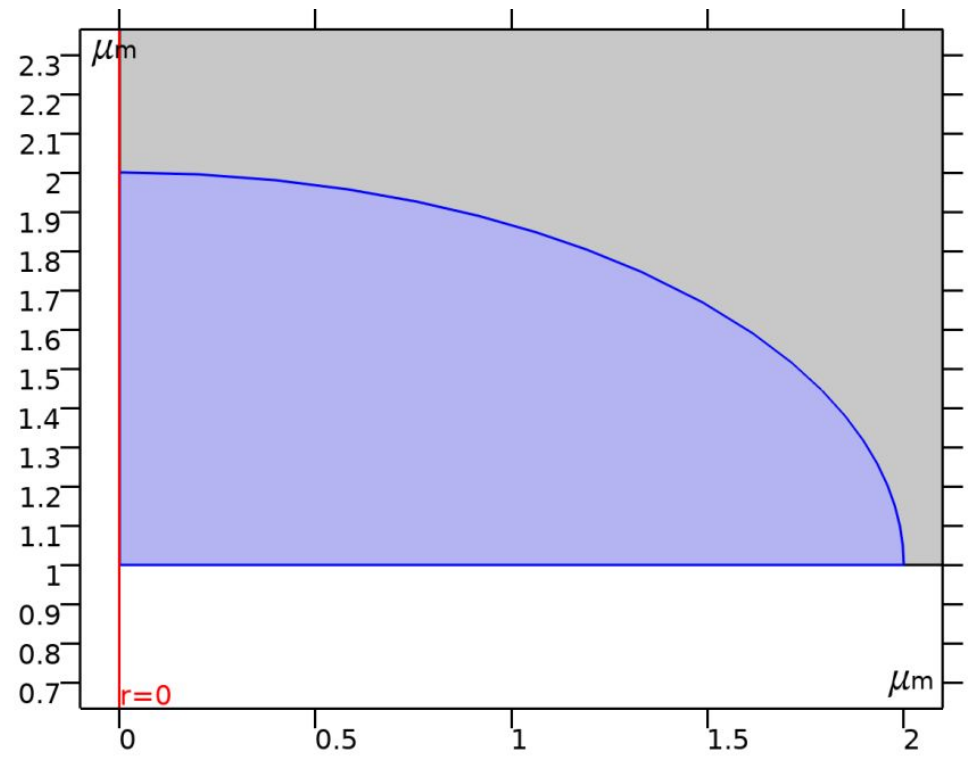

Transport of Diluted Species Droplet

EQUATIONS

$$
\begin{aligned}
& \frac{\partial c_{i}}{\partial t}+\nabla \cdot \mathbf{J}_{i}=R_{i} \\
& \mathbf{J}_{i}=-D_{i} \nabla c_{i}
\end{aligned}
$$

\section{FEATURES}

Transport Properties droplet

Axial Symmetry 1

No Flux 1

Initial Values 1 
Org Electrode flux

Flux_phase transfer

Reactions 1

\subsubsection{Transport Properties droplet}

EQUATIONS

$$
\begin{aligned}
& \frac{\partial c_{i}}{\partial t}+\nabla \cdot \mathbf{J}_{i}=R_{i} \\
& . \ldots \ldots \ldots \ldots . . \\
& \mathbf{J}_{i}=-D_{i} \nabla c_{i}
\end{aligned}
$$

\subsubsection{No Flux 1}

EQUATIONS

$$
-\mathbf{n} \cdot \mathbf{J}_{i}=0
$$

\subsubsection{Org Electrode flux}

EQUATIONS

$-\mathbf{n} \cdot \mathbf{J}_{i}=\jmath_{0, j}$

\subsubsection{Flux_phase transfer}

EQUATIONS

$$
-\mathbf{n} \cdot J_{i}=\jmath_{0, j} .
$$

\subsubsection{Reactions 1}

EQUATIONS

$$
\frac{\partial c_{i}}{\partial t}+\nabla \cdot \mathbf{J}_{i}=R_{i}+S_{i}
$$




\subsection{MESH 1}

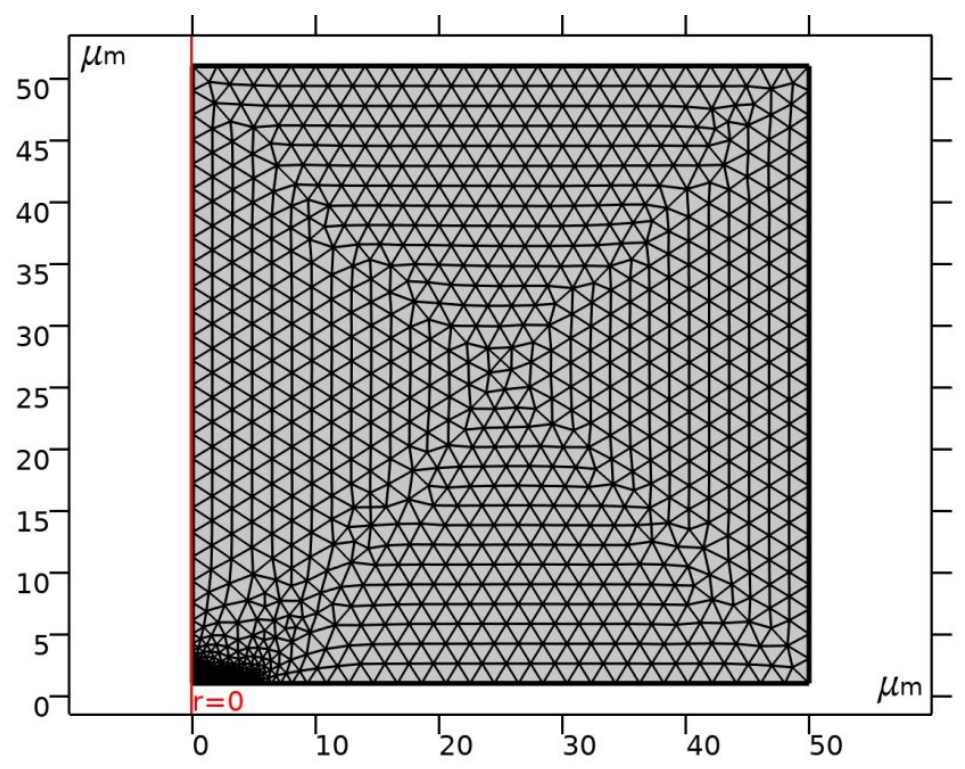

Mesh 1 


\section{Study 1}

COMPUTATION INFORMATION

\begin{tabular}{l|l} 
Computation time & $47 \mathrm{~s}$ \\
\hline CPU & Intel64 Family 6 Model 158 Stepping 10, 6 cores \\
\hline Operating system & Windows 10
\end{tabular}

\subsection{TIME DEPENDENT}

\begin{tabular}{|l|l|}
\hline Times & Unit \\
\hline range $(0,0.0001,0.5)$ & $\mathrm{s}$ \\
\hline
\end{tabular}

STUDY SETTINGS

\begin{tabular}{|l|l|}
\hline Description & Value \\
\hline Include geometric nonlinearity & Off \\
\hline
\end{tabular}

PHYSICS AND VARIABLES SELECTION

\begin{tabular}{|l|l|}
\hline Physics interface & Discretization \\
\hline Electroanalysis (elecDrop) & physics \\
\hline Electroanalysis (ElecAq) & physics \\
\hline Transport of Diluted Species Aqueous (tds_Aq) & physics \\
\hline Transport of Diluted Species Droplet (tds) & physics \\
\hline
\end{tabular}

MESH SELECTION

\begin{tabular}{|l|l|}
\hline Geometry & Mesh \\
\hline Geometry 1 (geom1) & mesh1 \\
\hline
\end{tabular}




\section{Results}

\subsection{DATASETS}

\subsubsection{Study $1 /$ Solution 1}

SOLUTION
\begin{tabular}{|l|l|}
\hline Description & Value \\
\hline Solution & Solution 1 \\
\hline Component & Save Point Geometry 1 \\
\hline
\end{tabular}

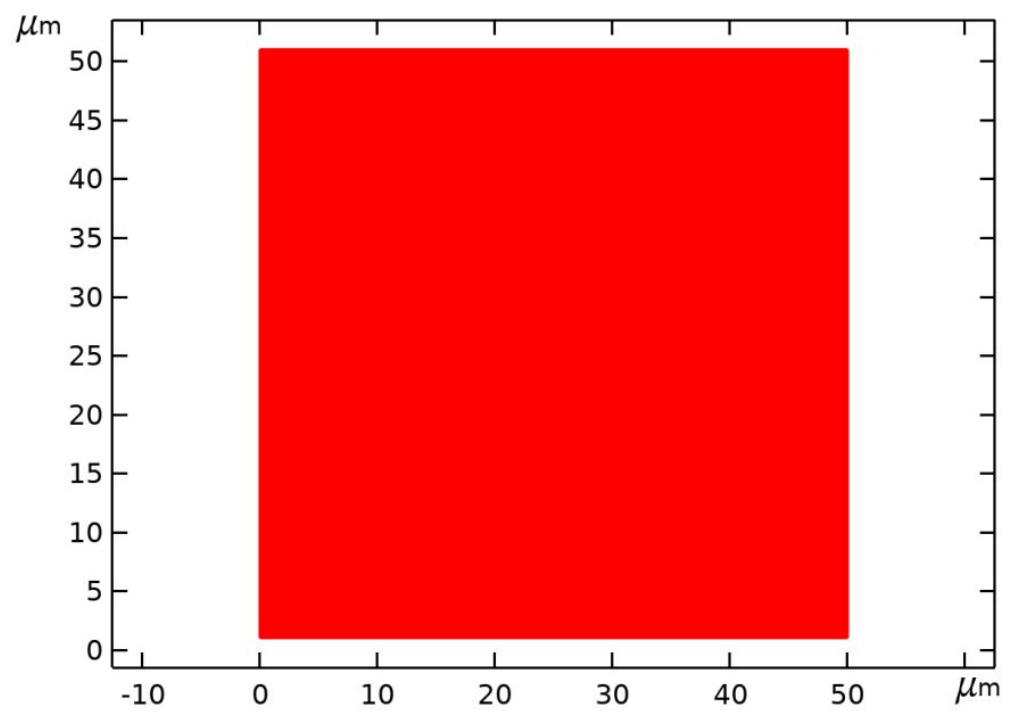

Dataset: Study 1/Solution 1

\subsection{DERIVED VALUES}

\subsubsection{Line Integration 1}

DATA

Description Value

Dataset Study $1 /$ Solution 1

EXPRESSIONS

\begin{tabular}{|l|l|l|}
\hline Expression & Unit & Description \\
\hline tds.ntflux_Cp*F_const & $A$ & \\
\hline
\end{tabular}

INTEGRATION SETTINGS

\begin{tabular}{|l|l|}
\hline Description & Value \\
\hline Integration order & 4 \\
\hline
\end{tabular}




\begin{tabular}{|l|l|}
\hline Description & Value \\
\hline Compute surface integral & On \\
\hline
\end{tabular}

\subsubsection{Surface Average 1}

DATA

\section{Description Value}

Dataset $\quad$ Study $1 /$ Solution 1

\section{EXPRESSIONS}

\begin{tabular}{|l|l|l|}
\hline Expression & Unit & Description \\
\hline $\mathrm{Cp}$ & $\mathrm{mol} / \mathrm{m}^{\wedge} 3$ & Concentration \\
\hline
\end{tabular}

INTEGRATION SETTINGS

\begin{tabular}{|l|l|}
\hline Description & Value \\
\hline Integration order & 4 \\
\hline Compute volume integral & On \\
\hline
\end{tabular}

\subsection{PLOT GROUPS}

\subsubsection{D Plot Group 6}

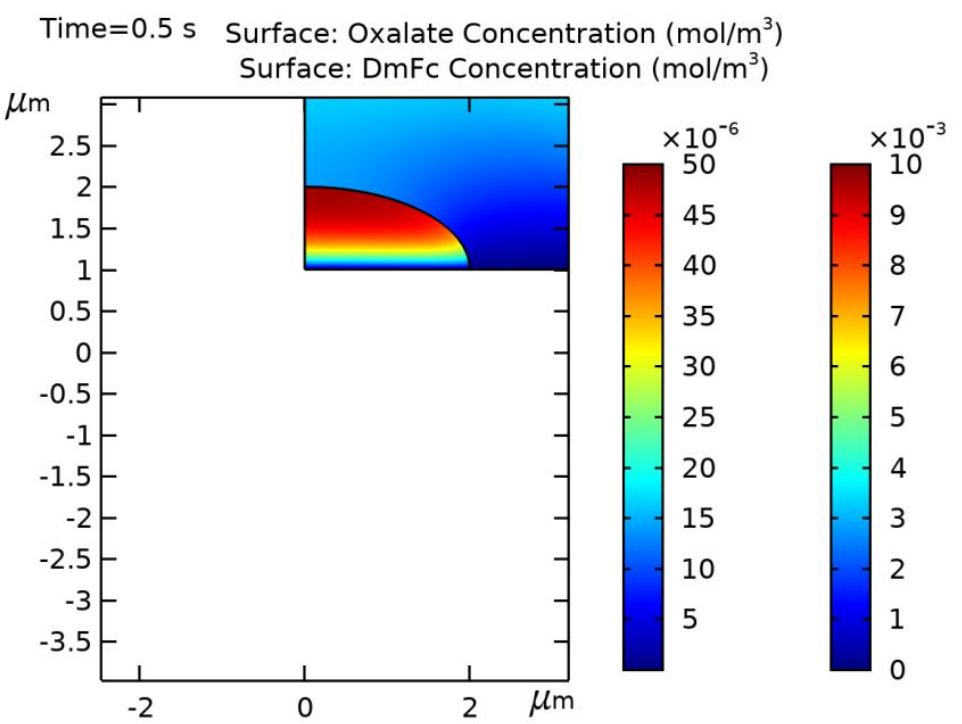

Surface: Oxalate Concentration $\left(\mathrm{mol} / \mathrm{m}^{3}\right)$ Surface: DmFc Concentration $\left(\mathrm{mol} / \mathrm{m}^{3}\right)$ 